\title{
INFLUÊNCIA DA CONDIÇÃO BUCAL NA QUALIDADE DE VIDA DE ADOLESCENTES: UMA REVISÃO INTEGRATIVA
}

\author{
Raphael Lobo de Souza $^{1}$; Ana Rita Duarte Guimarães ${ }^{2}$ \\ 1. Raphael Lôbo de Souzal PROBIC/UEFS, Graduando em Odontologia, Universidade Estadual de Feira de Santana, \\ e-mail:rapha_lobo_2011@hotmail.com \\ 2. Orientadora, Departamento de Saúde, Universidade Estadual de Feira de Santana, e-mail: ardg1999@gmail.com
}

PALAVRAS-CHAVE: Ansiedade Odontológica; Qualidade de vida; Adolescentes.

\section{INTRODUÇÃO}

Segundo a Organização Mundial de Saúde (OMS), pode-se considerar a adolescência como o período delimitado entre 10 e 19 anos de idade (OMS, 1965), ou seja, a segunda década de vida, sendo que mais de 34 milhões de brasileiros tinham entre 10 e 19 anos de idade no ano 2000 (BRASIL, 2007). A saúde bucal constitui um fator fundamental na autoimagem de cada adolescente, uma vez comprometida, afeta a saúde em geral, podendo alterar hábitos alimentares, dificuldades na mastigação, na fala, e conduzir mudanças no comportamento, insatisfação com a aparência e prejuízo na aceitação social, cujas implicações negativas poderão assumir um grande impacto na qualidade de vida destes. (SHEIHAM, 2005). Assim sendo, o objetivo deste estudo é reunir o conhecimento científico disponível atualmente na literatura sobre a associação entre o status de condição bucal de adolescentes e a qualidade de vida relatada por eles.

\section{METODOLOGIA}

O presente estudo configura-se como uma revisão integrativa, tipo de revisão da literatura onde foram utilizados dados de fontes primárias, por meio de um levantamento de trabalhos na literatura existente. O plano sistemático para a execução desta revisão integrativa consistiu em quatro etapas. Na primeira etapa, realizou-se o levantamento bibliográfico nas seguintes bases de dados: Lilacs (Literatura Latino-Americana e do Caribe em Ciências de Saúde) e Medline/Pubmed (Medical Literature Analysis and Retrieval System Online). Nesse sentido, aplicou-se como critérios de busca, os periódicos publicados no período de 2015 e 2016, utilizando a combinação das seguintes palavras-chave: qualidade de vida, saúde bucal, ansiedade odontológica e adolescentes, adequando o idioma de acordo com a base de dados utilizada. Dentre os periódicos encontrados, apenas aqueles que haviam sido publicados na íntegra foram avaliados na etapa seguinte da revisão.

A segunda etapa constituiu-se pela leitura dos resumos constantes dos artigos e documentos previamente selecionados e aplicando os critérios de inclusão.

$\mathrm{Na}$ terceira etapa, os estudos que atenderam os critérios previamente estabelecidos foram selecionados, e lidos na íntegra. Para auxiliar o desenvolvimento desta etapa foi criada uma planilha do programa Excel for Windows discriminando para cada artigo incluído nesse estudo (TABELA 1).

Para a categorização dos estudos foi utilizado à pirâmide de evidência de Savi (2009), uma versão traduzida da pirâmide de evidência de Suny (2001).

A quarta etapa seguiu-se a análise e interpretação dos dados que foram conduzidas buscando explicações em cada estudo para as variações nos resultados encontrados.

\section{RESULTADOS}


Foram revisadas 21 referências para este estudo, sendo a base de dados com maior número de artigos sobre esse tema foi o Pubmed, com 17 publicações, seguindo o Lilacs, com quatro artigos científicos encontrados. Destes, quatro não disponibilizavam o texto na íntegra gratuitamente na plataforma Capes, reduzindo assim para 17 o número de artigos selecionados. Em seguida, foi realizada a leitura dos periódicos, sendo eliminadas quatro publicações seguindo os critérios de inclusão descritos. Dessa forma, desta analise foram selecionadas 13 publicações.

Para a classificação quanto ao Nível de Evidência Científica por Tipo de Estudo, foi utilizado à classificação de Savi (2009) que se verificou o nível de evidência II correspondendo a um estudo (18), um estudo (22) no nível de evidencia III, um estudo encontrado no nível de evidência IV (25), nove estudos $(14,15,16,19,20,23,26)$ consistiu em nível de evidência $\mathrm{V}$. Um dos estudos selecionados não se encaixavam nesta classificação (17) por se tratar de um artigo sobre teste de validação de um instrumento para avaliar o impacto psicossocial da estética dentária em adultos jovens.

Para classificar o nível de indexação da revista, foi utilizada a Classificação Qualis 2014 da Capes a partir da plataforma online que permite relaciona-lo pelo nome da revista. Dos 13 artigos, três $(18,21,23)$ foram publicados em revistas nível A1, um (25) em nível A2, três $(15,20,26)$ foi nível B1, um (24) em nível B2, um (17) no B3, um foi B4 (14) e três artigos $(16,19,22)$ foram publicados em periódicos que não constavam nessa classificação pelo sistema utilizado.

Após a leitura dos artigos selecionados foi possível agrupá-los de acordo com o seu assunto principal. O Oral Health Impact Profile (OHIP-14) é o instrumento mais reprodutível ao descrever a qualidade de vida relacionada à saúde bucal em adolescentes $(14,19,21,22,26)$.

\section{CONSIDERAÇÕES FINAIS}

Neste estudo, de acordo com os periódicos estudados, a literatura é inconclusiva entre à associação da saúde bucal e qualidade de vida dos adolescentes. Assim, a percepção dos adolescentes em relação aos impactos odontológicos no desempenho de suas atividades diárias apresentou-se baixa. Além disso, são necessários estudos com períodos de acompanhamento longo e com amostras maiores, que ajudarão avaliar o impactado destes na qualidade de vida dos adolescentes.

\section{REFERÊNCIAS}

ORGANIZACIÓN MUNDIAL DE LA SALUD (OMS). Problemas de salud de la adolescência. Genebra: Série de informes técnicos 1965; 308: 29.

BRASIL. Ministério do Planejamento, Orçamento e Gestão. Estatísticas do século XX. Rio de Janeiro: IBGE, 2007. Diversas tabelas. Acesso em: 17/12/2010.

SHEIHAM A. Oral health, general health and quality of life. Bulletin of the World Health Organization 2005; 83(9): 641-720.

SAVI MGM, SILVA EL. O fluxo da informação na prática clínica dos médicos residentes: análise na perspectiva da medicina baseada em evidências. Ciência da Informação 2009;38(3):177-91.

SUNY DOWNSTATE MEDICAL CENTER. Evidence Based Medicine Course Evidence pyramid 2001.

PAREDES AO, JÚNIOR OSL, FERNANDES JMFA, MENEZES VA. Influencia da Saúde Bucal sobre a Qualidade de vida de Adolescentes Escolares. Rev Bras Promoç Saúde 2015; 28(2): 266-73.

LEÃO MM, GARBIN CAS, MOIMAZ SAS, ROVIDA TAS. Oral health and quality of life: an epidemiological survey of adolescents from settlement in Pontal do Paranapanema/SP, Brazil. Ciência \& Saúde Coletiva 2015; 20(11):3365-74 
KUMAR S, BADIYANI BK, KUMAR A, DIXIT G, SHARMA P, AGRAWAL S. Orofacial pain and quality of life in early adolescents in India. Int J Adolesc Med Health 2016

SANTOS PML, GONÇALVES AR, MAREGA T. Validity of the Psychosocial Impact of Dental Aesthetics Questionnaire for use on Brazilian adolescents. Dental Press J Orthod 2016; 21(3): 67-72.

AIMÉE NR, VAN WIJK AJ, MALTZ M, VARJÃO MM. MESTRINHO HD, CARVALHO JC. Dental caries, fluorosis, oral health determinants, and quality of life in adolescents. Clin Oral Invest 2016; 21(5): 1811-20.

XAVIER A, CARVALHO ES, BASTOS RS, CALDANA ML, DAMIANCE PRM, BASTOS JRM. Impact of dental caries on quality of life of adolescents according to access to oral health services: a cross sectional study. Braz J Oral Sci 2015; 15(1): 1-7

WRIGH WG, SPIRO A, JONES JA, RICH SE, GARCIA RI. DEVELOPMENT of the Teen Oral Health-Related Quality of Life Instrument. J Public Health Dent 2016; 77(2): 115- 24.

SILVA LFG, THOMAZ EBAF, FREITAS HV, PEREIRA ALP, RIBEIRO CCC, ALVES

CMC. Impact of Malocclusion on the Quality of Life of Brazilian Adolescents: A Population-Based Study. Plos One 2016; 11(9): e0162715.

ZHENG D ET AL. Assessing changes in quality of life using the Oral Health Impact Profile (OHIP) in patients with different classifications of malocclusion during comprehensive orthodontic treatment. BMC Oral Health 2015; 15:148.

ABREU LG, MELGACÔ CA, ABREU MHNG, LAGES EMB, PAIVA SM. Agreement Between Adolescents and Parents or Caregivers in Rating Adolescents' Quality of Life During Orthodontic Treatment. Am J Orthod Dentofacial Orthop 2015; 148(6): 1036-42.

TWIGGE E, ROBERTS RM, JAMIESON L, DREYER CW, WAYNE J. SAMPSON WJ. The psycho-social impact of malocclusions and treatment expectations of adolescent orthodontic patients. Eur J Orthod 2016;38(6):593-601.

COSTA AA; SERRA-NEGRA JM; BENDO CB; PORDEUS IA; PAIVA SM. Impact of wearing fixed orthodontic appliances on quality of life among adolescents: Case-control study. Angle Orthod 2016; 86(1):121-6.

OLIVEIRA DC, FERREIRA FM, MOROSINI IAC, TORRES-PEREIRA CC, PAIVA SM, FRAIZ FC. Impacto f Oral Health Status on The Oral Healt-Related Quality of Life of Brazilian Male Incarcerated Adolescents. Oral Health Prev Dent 2015;13(5): 417-25.

SLADE G. Derivation and validation of a short-form oral health impact profile. Community Dent Oral Epidemiol 1997; 25: 284-90 
Tabela 1. Estudos Selecionados

\begin{tabular}{|c|c|c|c|c|}
\hline Autor, Ano, País, Tipo de estudo & Amostra & $\begin{array}{c}\text { Instrumento } \\
\text { Qualidade de vida x Saúde } \\
\text { Bucal }\end{array}$ & $\begin{array}{l}\text { Nível de } \\
\text { Evidência }\end{array}$ & $\begin{array}{l}\text { Nível Qualis } \\
\quad 2014\end{array}$ \\
\hline $\begin{array}{c}\text { Paredes SO, Júnior OSL, } \\
\text { Paredes AO, Fernandes JMFA, Menezes } \\
\text { VA. (2015), P, O.T }\end{array}$ & $\begin{array}{l}184 \text { adolescentes } \\
\text { (15 a } 19 \text { anos). }\end{array}$ & $\begin{array}{l}\text { Perfil de Impacto na Saúde } \\
\text { Bucal (OHIP-14). }\end{array}$ & N.E. $\mathrm{V}$ & B4 \\
\hline $\begin{array}{l}\text { Leão MM, Garbin CAS, Moimaz SAS, } \\
\text { Rovida TAS. (2015), Brasil, O.T }\end{array}$ & $\begin{array}{l}180 \text { adolescentes } \\
(10 \text { a } 19 \text { anos })\end{array}$ & $\begin{array}{l}\text { Índice de Impactos Diários } \\
\text { sobre o Desempenho Diário } \\
\text { (OIDP-12) }\end{array}$ & N.E. $\mathrm{V}$ & B1 \\
\hline $\begin{array}{c}\text { Kumar S*, Badiyani BK, Kumar A, } \\
\text { Dixit G, Sharma P, Agrawal S. India, } \\
\text { 2016, O.T }\end{array}$ & $\begin{array}{l}800 \text { adolescentes } \\
(12-15 \text { anos })\end{array}$ & $\begin{array}{l}\text { Questionário Geral de Saúde } \\
\text { (GHQ-12) }\end{array}$ & N.E. $\mathrm{V}$ & - \\
\hline $\begin{array}{c}\text { Santos PM1, Gonçalves AR, Marega T. } \\
\text { Brasil, 2016, SC }\end{array}$ & $\begin{array}{l}194 \text { adolescentes } \\
(11-14 \text { anos })\end{array}$ & $\begin{array}{l}\text { Questionário de Impacto } \\
\text { Psicossocial da Estética } \\
\text { Dentária (PIDAQ) }\end{array}$ & - & B3 \\
\hline $\begin{array}{c}\text { Aimée NR,. Van Wijk AJ, Maltz M, M. } \\
\text { Varjão H. Mestrinho D, Carvalho JC. } \\
\text { (2016), PAIS, O.L }\end{array}$ & $\begin{array}{r}618 \text { indivíduos } \\
\text { (10 a } 15 \text { anos) }\end{array}$ & $\begin{array}{l}\text { Questionário de Percepção da } \\
\text { Criança (CPQ11-14) }\end{array}$ & N.E. II & A1 \\
\hline $\begin{array}{c}\text { Xavier A, Carvalho ES, Bastos RS, } \\
\text { Caldana ML, Damiance PRM, Bastos } \\
\text { JRM. (2016), Brasil, O.T }\end{array}$ & $\begin{array}{l}526 \text { adolescentes } \\
(15 \text { e } 19 \text { anos })\end{array}$ & $\begin{array}{l}\text { Perfil de Impacto na Saúde } \\
\text { Bucal (OHIP-14). }\end{array}$ & N.E. $\mathrm{V}$ & - \\
\hline $\begin{array}{c}\text { Wrigh WG, Spiro A, Jones JA, Rich } \\
\text { SE, Garcia RI } \\
(2016) \text {, EUA, O.T. }\end{array}$ & $\begin{array}{l}363 \text { adolescentes } \\
(13 \text { e } 18 \text { anos })\end{array}$ & $\begin{array}{l}\text { Qualidade de vida } \\
\text { relacionada à saúde bucal dos } \\
\text { adolescentes (TOQOL) }\end{array}$ & N.E. $\mathrm{V}$ & B1 \\
\hline $\begin{array}{l}\text { Silva LFG, Thomaz EBAF, Freitas HV, } \\
\text { Pereira ALP, Ribeiro CCC, Alves CMC. } \\
\text { (2016), Brasil, O.T }\end{array}$ & $\begin{array}{l}1015 \text { adolescentes } \\
(12 \text { a } 15 \text { anos })\end{array}$ & $\begin{array}{c}\text { Perfil de Impacto na Saúde } \\
\text { Bucal (OHIP-14) e o DAÍ } \\
\text { (INDICE ESTÉTICO } \\
\text { DENTÁRIO) } \\
\end{array}$ & N.E. $\mathrm{V}$ & A1 \\
\hline Zheng D et al.( 2015 ), China, Coorte, & $\begin{array}{l}81 \text { pacientes } \\
(15 \text { a } 24 \text { anos })\end{array}$ & $\begin{array}{l}\text { Perfil de Impacto na Saúde } \\
\text { Bucal (OHIP-14). }\end{array}$ & N.E. III & - \\
\hline $\begin{array}{c}\text { Abreu LG, Melgacô CA, Abreu MHNG, } \\
\text { Lages EMB, Paiva SM. (2015), Brasil, } \\
\text { O.T }\end{array}$ & $\begin{array}{l}102 \text { pares de pais } \\
\text { adolescentes / } \\
\text { cuidadores } \\
(11 \text { e } 12 \text { anos })\end{array}$ & $\begin{array}{l}\text { Os adolescentes responderam } \\
\text { à forma abreviada do Child } \\
\text { Perceptions Questionnaire } \\
\text { (CPQ11-14) e os pais } \\
\text { Questionário de Percepção } \\
\text { Parental-Cuidador (PCPQ). }\end{array}$ & N.E. V & A1 \\
\hline $\begin{array}{c}\text { Twigge E, Roberts RM, Jamieson L, } \\
\text { Dreyer CW, Wayne J. Sampson WJ. } \\
\text { (2016), Australia, O.T }\end{array}$ & $\begin{array}{l}105 \text { Adolescentes } \\
\text { (12 e } 17 \text { anos })\end{array}$ & $\begin{array}{c}\text { Questionário de Impacto } \\
\text { Psicossocial da Estética } \\
\text { Dentária (PIDAQ) e a } \\
\text { avaliação do Impacto Oral na } \\
\text { Performance Diária (OIDP) }\end{array}$ & N.E. $\mathrm{V}$ & B2 \\
\hline $\begin{array}{c}\text { Costa AA; Serra-Negra JM; Bendo CB; } \\
\text { Pordeus IA; Paiva SM. (2015), Brasil, } \\
\text { C.C }\end{array}$ & $\begin{array}{l}327 \text { adolescentes } \\
\text { (11 a } 14 \text { anos) }\end{array}$ & $\begin{array}{c}\text { Child Perceptions } \\
\text { Questionnaire (CPQ11-14) }\end{array}$ & N.E. IV & A2 \\
\hline $\begin{array}{c}\text { Oliveira DC, Ferreira FM, Morosini } \\
\text { IAC, Torres-Pereira CC, Paiva SM e } \\
\text { Fraiz FC. (2015), Brasil, O.T }\end{array}$ & 102 adolescentes & $\begin{array}{l}\text { Perfil de Impacto na Saúde } \\
\text { Bucal (OHIP-14) }\end{array}$ & N.E. $\mathrm{V}$ & B1 \\
\hline
\end{tabular}

Legenda: O. T.= Observacional Transversal; O. L.= Observacional Longitudinal; C.C= Caso Controle; S.C= Sem Classificação 